\title{
A SIMILARITY SOLUTION OF FIN EQUATION WITH VARIABLE THERMAL CONDUCTIVITY AND HEAT TRANSFER COEFFICIENT
}

\author{
M. Pakdemirli* and A. Z. Şahin** \\ * Department of Mechanical Engineering, Celal Bayar University, \\ 45140 Muradiye, Manisa, Turkey \\ **Department of Mechanical Engineering, King Fahd University of Petroleum and \\ Minerals, Dhahran 31261, Saudi Arabia
}

\begin{abstract}
A nonlinear fin equation in which thermal conductivity is an arbitrary function of temperature and, heat transfer coefficient is an arbitrary function of spatial variable is considered. Scaling transformation is applied to the equations to determine the specific forms of these functions for which the equation admits such type of transformation. It is found that for arbitrary heat conduction function, scaling transformation exists for an inverse square heat transfer coefficient. Selecting also the conductivity as an exponential function, the partial differential equation is transferred to an ordinary differential equation via similarity transformations. The resulting equation is solved numerically and temperature distribution is determined for various heat conductivity parameters.
\end{abstract}

\section{INTRODUCTION}

Heat transfer through extended surfaces has been studied extensively in the literature. Constant thermo-physical properties and uniform heat transfer coefficient is often assumed in the determination of the temperature distribution along an extended surface. The mathematical complexity of the conservation energy equation is reduced by this assumption and therefore well-established closed form analytical solution can be obtained for a number of cases. However, this assumption will lead to poor prediction of the thermal performance of the extended surface especially for certain fin geometries.

Nnanna et al. [1] developed a generalized analytical solution for the computation of heat loss from an extended surface based on variable heat transfer coefficient, fin geometry and fin surface curvature. Lee et al. [2] applied a hybrid numerical method of the Laplace transformation and the finite difference method to solve the transient thermo-elastic problem of an annular fin assuming the heat transfer coefficient is a function of the radius of the fin. Yang and He [3] solved the problem of one dimensional, transient heat transfer in a fin by the method of small parameter perturbation and by reducing the partial differential equation to a system of ordinary differential equations. They used shooting and superposition method to solve the system of ordinary differential equations. Chu and Chang [4] used a hybrid numerical technique to investigate a two-dimensional cylindrical pin fin with arbitrary variable Biot numbers on the fin lateral and tip surfaces. Yu and Chen [5] discussed the optimization of rectangular profile circular fins with variable thermal conductivity and convective heat transfer coefficients. They used differential transformation method to solve the nonlinear heat transfer equation. Natarajan and Shenoy [6] used the principles of variational calculus to determine the shapes of convective pin fins that maximize heat 
dissipation for fixed amount of fin material. They considered the convective heat transfer coefficient to vary with the fin diameter according to a power law. Zubair et al. [7] obtained the optimal dimensions of circular fins with variable profile and temperature-dependent thermal conductivity. Chiu and Chen [8] used the Adomian decomposition method to evaluate the efficiency and the optimal length of a convective rectangular fin with variable thermal conductivity and to determine the temperature distribution within the fin in the form of infinite power series.

Very limited amount of work can be found on the similarity solution to heat transfer problems through extended surfaces especially with variable thermal properties. Campo and Salazar [9] studied the analogy between unsteady-state conduction in a planar slab for short times and steady-state conduction in a straight fin of uniform cross section. They also presented approximate analytical solutions of the transient heat conduction equation for short times in a plane having a uniform initial temperature and subjected to a uniform surface temperature. Kuehn et al. [10] studied the similarity solution or conjugate natural convection heat transfer from a long vertical plate fin. They presented complete result for a uniform conductivity plate fin as a function of the fluid Prandtl number. In the present study, a similarity solution is attempted to the nonlinear fin equation in which the thermal conductivity is an arbitrary function of temperature and heat transfer coefficient is an arbitrary function of spatial variable. The types of functions for the thermal conductivity and the heat transfer coefficient for which similarity solutions could be obtained were investigated.

\section{THEORETICAL ANALYSIS FOR SCALING TRANSFORMATION}

Following a similar approach as presented in reference [11], the dimensionless, nonlinear fin equation can be written as,

$$
\frac{\partial}{\partial \mathrm{x}}\left[\mathrm{k}(\theta) \frac{\partial \theta}{\partial \mathrm{x}}\right]-\mathrm{N}^{2} \mathrm{f}(\mathrm{x}) \theta=\frac{\partial \theta}{\partial \mathrm{t}}
$$

where $\theta$ is the dimensionless temperature, $\mathrm{x}$ and $\mathrm{t}$ are the dimensionless spatial and time variables respectively. Thermal conductivity is an arbitrary function of temperature and heat transfer coefficient is an arbitrary function of the spatial variable. $\mathrm{N}$ is the fin parameter. In [11] a linear thermal conductivity is assumed. Our aim in this study is to apply scaling transformation to the equation and determine the specific forms of $k(\theta)$ and $f(x)$ for which the fin equation admits this transformation. For applications of scaling symmetries as well as other types of special Lie Group transformations, and determining specific forms of functions for which these symmetries exist, see [12-16] for example. In the degenerate case of equation (1) with $f(x)=0$, a complete group classification has been presented previously [17]. The complete group classification of the more general case of $f(x) \neq 0$ has been treated only recently [18].

The fin equation should be invariant under the below scaling transformations

$$
\overline{\mathrm{x}}=\lambda^{\mathrm{a}} \mathrm{x}, \quad \overline{\mathrm{t}}=\lambda^{\mathrm{b}} \mathrm{t}, \quad \bar{\theta}=\lambda^{\mathrm{c}} \theta
$$

which leads to the following invariance conditions

$$
\begin{aligned}
& \lambda^{2 \mathrm{a}-\mathrm{b}} \mathrm{k}\left(\lambda^{-\mathrm{c}} \bar{\theta}\right)=\mathrm{k}(\theta) \\
& \lambda^{-\mathrm{b}} \mathrm{f}\left(\lambda^{-\mathrm{a}} \overline{\mathrm{x}}\right)=\mathrm{f}(\mathrm{x})
\end{aligned}
$$


To determine the specific forms of these functions, equations are differentiated with respect to $\lambda$ and expressed in terms of original variables

$$
\begin{aligned}
& (2 \mathrm{a}-\mathrm{b}) \mathrm{k}(\theta)-\mathrm{c} \theta \mathrm{k}^{\prime}(\theta)=0 \\
& \mathrm{bf}(\mathrm{x})+\mathrm{ax} \mathrm{f}^{\prime}(\mathrm{x})=0
\end{aligned}
$$

Equation (5) leads to three distinct cases:

\section{i. $\mathbf{k}=\mathbf{k}(\theta)$}

For this case, the thermal conductivity is an arbitrary function of temperature which requires the coefficients of equation (5) to vanish and hence $b=2 a$ and $c=0$. If the heat transfer coefficient is required to be an arbitrary function of $x$, from equation (6) $\mathrm{a}=\mathrm{b}=0$ which means that the fin equation does not accept scaling transformation when both functions are arbitrary. Selecting $f(x)=1$ does not improve the situation. However, solving (6) for $\mathrm{f}(\mathrm{x})$ with $\mathrm{b}=2 \mathrm{a}$ yields $\mathrm{f}(\mathrm{x})=1 / \mathrm{x}^{2}$. For this special choice, the parameters are

$$
\mathrm{b}=2 \mathrm{a}, \quad \mathrm{c}=0 \quad \text { for } \mathrm{k}=\mathrm{k}(\theta) \text { and } \mathrm{f}=1 / \mathrm{x}^{2}
$$

Note that the constant appearing in the solution of $f(x)$ can be embedded into the fin parameter $\mathrm{N}$.

ii. $\mathbf{k}=\mathbf{k}_{\mathbf{0}}$

For this case, the thermal conductivity is constant. From equation (5), c is arbitrary and $b=2 a$. Requiring $f(x)$ to be arbitrary yields $a=b=0$ with no scaling transformations in the independent variables. For a specific form of $f(x)=1 / x^{2}$ however, the scaling symmetries increase

\section{iii. $\mathbf{k}=\mathbf{k}_{0} \underline{\underline{\theta^{\beta}}}$}

$$
\mathrm{b}=2 \mathrm{a}, \quad \mathrm{c} \text { arbitrary for } \mathrm{k}=\mathrm{k}_{0} \text { and } \mathrm{f}=1 / \mathrm{x}^{2}
$$

This case is obtained by integrating equation (5) directly. Here $\beta=(2 a-b) / c . f(x)$ cannot be arbitrary since $a=b=0$ from equation (6) which leads to $\beta=0$, the previous case. For $\mathrm{f}=1$, the symmetries are

$$
\mathrm{b}=0, \quad \mathrm{c}=2 \mathrm{a} / \beta \text { for } \mathrm{k}=\mathrm{k}_{0} \theta^{\beta} \text { and } \mathrm{f}=1
$$

The symmetries increase if equation (6) is integrated. The result is $f(x)=x^{m}$ where $m=-$ b/a. The symmetries are

$$
\mathrm{b}=-\mathrm{ma}, \quad \mathrm{c}=(2+\mathrm{m}) \mathrm{a} / \beta \quad \text { for } \mathrm{k}=\mathrm{k}_{0} \theta^{\beta} \text { and } \mathrm{f}=\mathrm{x}^{\mathrm{m}}
$$

\section{SIMILARITY TRANSFORMATION AND NUMERICAL RESULTS}

Numerical results will be produced for the following heat conductivity and heat transfer coefficients

$$
\mathrm{k}=\mathrm{k}_{0} \mathrm{e}^{\alpha \theta}, \quad \mathrm{h}=\frac{1}{\mathrm{x}^{2}}
$$

For this choice, one may use equation (7) for writing the determining equations for similarity variables

$$
\frac{\mathrm{dx}}{\mathrm{ax}}=\frac{\mathrm{dt}}{2 \mathrm{at}}=\frac{\mathrm{d} \theta}{0}
$$

Solving the system, one has the below similarity variable and function

$$
\mu=\frac{\mathrm{x}}{\sqrt{\mathrm{t}}}, \quad \theta=\theta(\mu)
$$


Substituting these variables into the original equation with the specific choice of $\mathrm{f}$ and $\mathrm{k}$ functions, one finally converts the partial differential equation into an ordinary differential equation

$$
\theta^{\prime \prime}+\frac{\mu}{2 \mathrm{k}_{0}} \mathrm{e}^{-\alpha \theta} \theta^{\prime}+\alpha \theta^{\prime 2}-\frac{\mathrm{N}^{2}}{\mu^{2} \mathrm{k}_{0}} \mathrm{e}^{-\alpha \theta} \theta=0
$$

The boundary conditions for the problem are

$$
\theta(0)=0, \quad \theta(\infty)=1
$$

Runge-Kutta algorithm Ode45 of MATLAB is used in numerical integrations. To satisfy the boundary condition at infinity, shooting technique is used. By trial and error, an appropriate $\theta^{\prime}(0)$ value is determined for which the condition at infinity is satisfied. To handle the singularity at $\mu=0$, the integration is started in a positive neighborhood of zero. If $\varepsilon<<1$ represents a very small positive value, the first condition can be transferred to the neighborhood value by Taylor series expansion

$$
\theta(\varepsilon)=\theta(0)+\varepsilon \theta^{\prime}(0)
$$

We selected numerical solutions which are independent of the selection of parameter $\varepsilon$. This can occur only for small fin parameters $\mathrm{N}$.

Figure 1 shows temperature profiles versus the similarity variable for different conductivity parameters $\alpha$.

For the same choice of parameters, the numerical integration is repeated for various $\varepsilon$ values. As can be seen from Figure 2, the temperature at infinity is almost independent of the choice of $\varepsilon$.

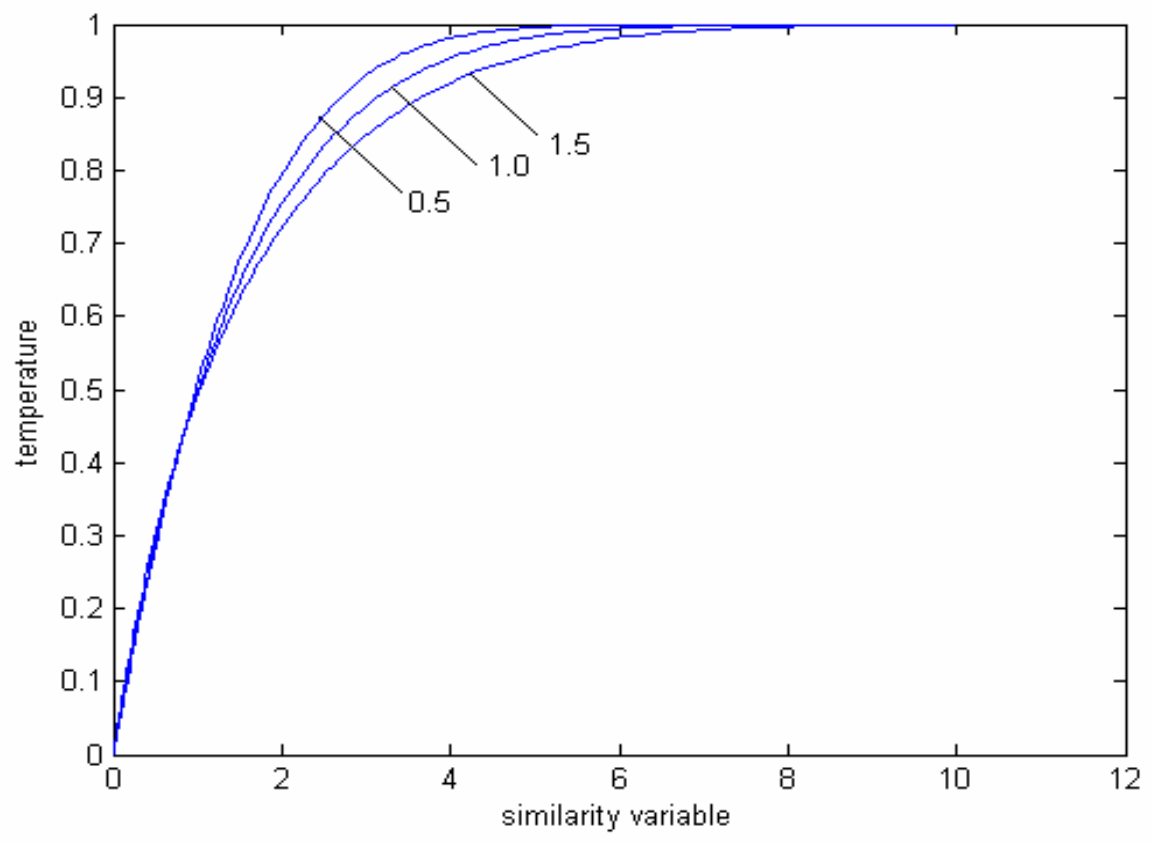

Figure 1-Temperature vs. similarity variable for various heat conductivity parameters

$$
\left(\mathrm{k}_{0}=1, \mathrm{~N}=0.02, \varepsilon=0.001\right)
$$




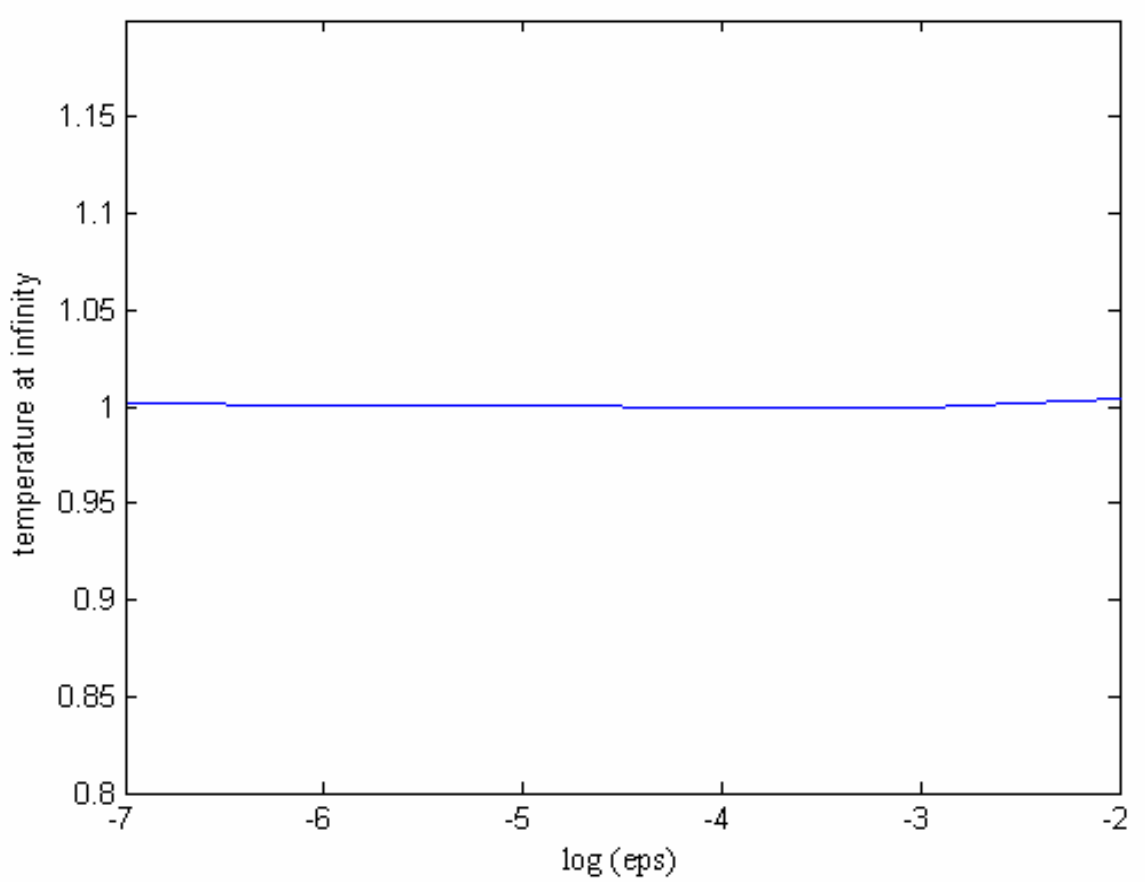

Figure 2- Temperature at infinity vs. logarithm of $\varepsilon\left(\mathrm{k}_{0}=1, \mathrm{~N}=0.02, \alpha=1\right)$

\section{CONCLUDING REMARKS}

A similarity analysis is presented for the fin equation with variable heat conduction and heat transfer coefficients. The specific forms of these functions are determined for equation to admit scaling symmetry. For the case of exponential temperature variation of heat conduction and inverse square spatial variation of heat transfer coefficients, fin equation is reduced to an ordinary differential equation via a similarity transformation. Resulting ordinary differential equation is integrated using a Runge-Kutta algorithm combined with shooting.

Acknowledgements- The authors acknowledge the support of King Fahd University of Petroleum \& Minerals, Dhahran, Saudi Arabia for this work.

\section{REFERENCES}

1. A. G. A. Nnanna, A. Haji-Sheikh and D. Agonafer, Effect of Variable Heat Transfer Coefficient, Fin Geometry, and Curvature on the Thermal Performance of Extended Surfaces, Journal of Electronic Packaging, Transactions of the ASME 125, 456-460, 2003.

2. H.-L. Lee, Y.-C. Yang and S.-S. Chu, Transient Thermoelastic Analysis of an Annular Fin with Coupling Effect and Variable Heat Transfer Coefficient, Journal of Thermal Stresses 25, 1105-1120, 2002.

3. X. Yang and H. He, Periodic heat transfer in the fins with variable thermal parameters, Applied Mathematics and Mechanics 17, 169-180, 1996. 
4. S.-S. Chu and W.-J. Chang, Hybrid Numerical Method for Transient Analysis of Two-Dimensional Pin Fins with Variable Heat Transfer Coefficients, International Communications in Heat and Mass Transfer 29, 367-376, 2002.

5. L.-T. Yu and C.-K. Chen, Optimization of Circular Fins with Variable Thermal Parameters, Journal of the Franklin Institute 336, 77-95, 1999.

6. U. Natarajan and U. V. Shenoy, Optimum shapes of convective pin fins with variable heat transfer coefficient, Journal of the Franklin Institute 327, 965-982, 1990.

7. S. M. Zubair, A.Z. Al-Garni and J.S. Nizami, The optimal dimensions of circular fins with variable profile and temperature-dependent thermal conductivity, International Journal of Heat and Mass Transfer 39, 3431-3439, 1996.

8. C.-H. Chiu and C.-K. Chen, A decomposition method for solving the convective longitudinal fins with variable thermal conductivity, International Journal of Heat and Mass Transfer 45, 2067-2075, 2002.

9. A. Campo and A. Salazar, Similarity between unsteady-state conduction in a planar slab for short times and steady-state conduction in a uniform, straight fin, Heat and Mass Transfer 31, 365-370, 1996.

10. T. H. Kuehn, S. S. Kwon and A. K. Tolpadi, Similarity Solution for Conjugate Natural Convection Heat Transfer from a Long Vertical Plate Fin, International Journal of Heat and Mass Transfer 26, 1718-1721, 1983.

11. A. Aziz and T. Y. Na, Periodic heat transfer in fins with variable thermal parameters, International Journal of Heat and Mass Transfer 24, 1397-1404, 1981.

12. A. G. Hansen and T. Y. Na, Similarity solutions of laminar, incompressible, boundary layer equations of non-Newtonian fluids, ASME Journal of Basic Engineering 90, 71-74, 1968.

13. M. G. Timol and N. L. Kalthia, Similarity solutions of three-dimensional boundary layer equations of non-Newtonian fluids, International Journal of Non-Linear Mechanics 21, 475-481, 1986.

14. M. Pakdemirli, Similarity analysis of boundary layer equations of a class of nonNewtonian fluids, International Journal of Non-Linear Mechanics 29, 187-196, 1994. 15. M. Pakdemirli and M. Yürüsoy, Similarity transformations for partial differential equations, SIAM Review 40, 96-101, 1998.

16. M. Pakdemirli, The boundary layer equations of third grade fluids, International Journal of Non-Linear Mechanics 27, 785-793, 1992.

17. G. W. Bluman and S. Kumei, Symmetries and Differential Equations, SpringerVerlag, New York, 1989.

18. M. Pakdemirli and A. Z. Şahin, Group classification of fin equation with variable thermal properties, International Journal of Engineering Science 42, 1875-1889, 2004. 